\title{
The Leech Method of Blood Analysis; A New Micro=Method*
}

\section{Determination of Blood Chlorine.}

By

MATSUITI YOSHIDA.

(吉 田松 一)

(From the Pediatric Department, Faculty of Medicine, Tohoku

Imperial University, Sendai. Director: Prof. A. Sato.)

The leech method has been successfully used for cholesterol, ${ }^{1)}$ potassium, ${ }^{2)}$ sodium," urea ${ }^{4)}$ and calcium. ${ }^{5)}$ The present paper is for blood chlorine.

Leeches which had been plump with sucked blood were fixed to a piece of wood with needles and the body was cut with scissors. The blood flowing out was received into a test tube for determination. The method used was that of Koranyi-Rusznák. ${ }^{6)}$

\section{Result.}

TABLE I.

Difference of blood chloride figures between the direct (auricular) method and the indirect (the leech method).

(0.1 c.c. blood used for each sample for the determination of $\mathrm{NaCl}$ )

\begin{tabular}{|c|c|c|c|c|c|}
\hline \multirow{2}{*}{$\begin{array}{l}\text { Nos, of rabbits } \\
\text { (body weight } \\
\text { in kilos) }\end{array}$} & \multirow{2}{*}{$\begin{array}{l}\text { Blood } \mathrm{NaCl} \text { in } \\
\text { rabbit's blood, } \\
\text { mgrms } \%\end{array}$} & \multicolumn{3}{|c|}{$\begin{array}{l}\text { Blood } \mathrm{NaCl} \text { in leech's blood, } \\
\text { mgrms } \%\end{array}$} & \multirow{2}{*}{$\begin{array}{l}\text { Difference } \\
(\%)\end{array}$} \\
\hline & & Nos. of leeches & Nos. of & mples & \\
\hline \multirow[b]{2}{*}{$\begin{array}{l}\text { No. } 1 \\
(2.50)\end{array}$} & \multirow[b]{2}{*}{567} & No. 1 & $\begin{array}{r}\text { No. } 1 \\
\text { No. } 2\end{array}$ & $\begin{array}{l}550 \\
546\end{array}$ & $\begin{array}{l}-3.1 \\
-3.7\end{array}$ \\
\hline & & No. 2 & $\begin{array}{l}\text { No. } 1 \\
\text { No. } 2 \\
\text { No. } 3\end{array}$ & $\begin{array}{l}549 \\
540 \\
550\end{array}$ & $\begin{array}{l}-3.2 \\
-\quad 4.8 \\
-\quad 3.1\end{array}$ \\
\hline
\end{tabular}

* 16th Report on Micro Method.

1) T. Sato, Tohoku J. Exp. Med., 1932, 19, 162.

2) Y. Ug a, Tohoku J. Exp. Med, 1932, 19, 165.

3) X.Uga, to be published in this journal.

4) M. Hasegawa, to be published later in this journal.

5) M. Yoshida, Tohoku J, Exp. Med., 1933, 22, 467.

6) L, Pincussen, Micromethodik, 1930 Leipzig. 


\begin{tabular}{|c|c|c|c|c|c|}
\hline \multirow{2}{*}{$\begin{array}{l}\text { Nos of rabbits } \\
\text { (body weight } \\
\text { in kilos) }\end{array}$} & \multirow{2}{*}{$\begin{array}{l}\text { Blood NaCl in } \\
\text { rabbit's blood, } \\
\text { mglms } \%\end{array}$} & \multicolumn{3}{|c|}{$\begin{array}{c}\text { Blood } \mathrm{NaCl} \text { in leech's blood, } \\
\text { mgrms } \%\end{array}$} & \multirow{2}{*}{$\begin{array}{c}\text { Difference } \\
(\%)\end{array}$} \\
\hline & & Nos. of leeches & Nos. of & nples & \\
\hline \multirow{2}{*}{$\begin{array}{l}\text { No. } 2 \\
(2.00)\end{array}$} & \multirow{2}{*}{546} & No. 3 & $\begin{array}{l}\text { No. } 1 \\
\text { No. } 2 \\
\text { No. } 3\end{array}$ & $\begin{array}{l}518 \\
459 \\
463\end{array}$ & $\begin{array}{r}-4.8 \\
-15.8 \\
-15.4\end{array}$ \\
\hline & & No. 4 & $\begin{array}{l}\text { No. } 1 \\
\text { No. } 2\end{array}$ & $\begin{array}{l}532 \\
537\end{array}$ & $\begin{array}{l}-2.7 \\
-1.8\end{array}$ \\
\hline \multirow{2}{*}{$\begin{array}{l}\text { No. } 3 \\
(1.77)\end{array}$} & \multirow[b]{2}{*}{547} & No. 5 & $\begin{array}{l}\text { No. } 1 \\
\text { No. } 2\end{array}$ & $\begin{array}{l}540 \\
535\end{array}$ & $\begin{array}{l}-1.3 \\
-\quad 2.2\end{array}$ \\
\hline & & No 6. & $\begin{array}{l}\text { No. } 1 \\
\text { No. } 2 \\
\text { No. } 3\end{array}$ & $\begin{array}{l}475 \\
475 \\
463\end{array}$ & $\begin{array}{l}-13.3 \\
-13.3 \\
-13.5\end{array}$ \\
\hline $\begin{array}{l}\text { No. } 4 \\
(2.45)\end{array}$ & 550 & No. 7 & $\begin{array}{l}\text { No. } 1 \\
\text { No. } 2 \\
\text { No. } 3\end{array}$ & $\begin{array}{l}510 \\
532 \\
531\end{array}$ & $\begin{array}{r}-7.3 \\
-\quad 3.3 \\
-\quad 3.5\end{array}$ \\
\hline & & & & rage & $-6.5 \%$ \\
\hline
\end{tabular}

TABLE II.

Difference of blood chloride figures between the direct (auricular) method and the indirect (the leech method).

$(0.13$ c.c. blood used for each sample for the determination of $\mathrm{NaCl})$

\begin{tabular}{|c|c|c|c|c|c|}
\hline \multirow{2}{*}{$\begin{array}{l}\text { Nos, of rabbits } \\
\text { (body weight } \\
\text { in kilos) }\end{array}$} & \multirow{2}{*}{$\begin{array}{l}\text { Blood NaCl in } \\
\text { rabbit's blood } \\
\text { (standard), } \\
\text { mgrms } \%\end{array}$} & \multicolumn{3}{|c|}{$\begin{array}{c}\text { Blood } \mathrm{NaCl} \text { in leech's blood, } \\
\text { mgrms } \%\end{array}$} & \multirow{3}{*}{$\begin{array}{l}\text { Difference } \\
\begin{array}{c}(\%) \\
-1.4 \\
-0.4 \\
-2.2\end{array}\end{array}$} \\
\hline & & Nos. of leeches & Nos. of & nples & \\
\hline \multirow{2}{*}{$\begin{array}{c}\text { No. } 1 \\
(2.685)\end{array}$} & \multirow{2}{*}{506} & No. 1 & $\begin{array}{l}\text { No. } 1 \\
\text { No. } 2 \\
\text { No. } 3\end{array}$ & $\begin{array}{l}499 \\
504 \\
495\end{array}$ & \\
\hline & & No. 2 & $\begin{array}{l}\text { No. } 1 \\
\text { No. } 2 \\
\text { No. } 3\end{array}$ & $\begin{array}{l}499 \\
509 \\
486\end{array}$ & $\begin{array}{ll}- & 1.4 \\
+ & 0.6^{*} \\
- & 3.9\end{array}$ \\
\hline $\begin{array}{c}\text { No. } 2 \\
(2.685)\end{array}$ & 486 & No. 3 & $\begin{array}{l}\text { No. } 1 \\
\text { No. } 2 \\
\text { No. } 3\end{array}$ & $\begin{array}{l}468 \\
482 \\
466\end{array}$ & $\begin{array}{r}-3.7 \\
-\quad 0.8 \\
-\quad 4.1\end{array}$ \\
\hline \multirow{3}{*}{$\begin{array}{c}\text { No. } 3 \\
(2.160)\end{array}$} & \multirow{3}{*}{483} & No. 4 & $\begin{array}{l}\text { No. } 1 \\
\text { No. } 2 \\
\text { No. } 3 \\
\text { No. } 4 \\
\text { No. } 5\end{array}$ & $\begin{array}{l}432 \\
468 \\
459 \\
462 \\
464\end{array}$ & $\begin{array}{r}-10.1 \\
-\quad 3.1 \\
-\quad 4.9 \\
-\quad 4.9 \\
-\quad 3.9\end{array}$ \\
\hline & & No. 5 & No. 1 & 464 & -3.9 \\
\hline & & No. 6 & $\begin{array}{l}\text { No. } 1 \\
\text { No. } 2 \\
\text { No. } 3\end{array}$ & $\begin{array}{l}473 \\
486 \\
468\end{array}$ & $\begin{array}{l}-2.1 \\
+\quad 0.6^{x} \\
-\quad 3.1\end{array}$ \\
\hline
\end{tabular}




\begin{tabular}{|c|c|c|c|c|c|}
\hline \multirow{2}{*}{$\begin{array}{l}\text { Nos. of rabbits } \\
\text { (body weight } \\
\text { in kilos) }\end{array}$} & \multirow{2}{*}{$\begin{array}{l}\text { Blood NaCl in } \\
\text { rabbit's blood } \\
\text { (standard) } \\
\text { mgrms } \%\end{array}$} & \multicolumn{3}{|c|}{$\begin{array}{l}\text { Blood } \mathrm{NaCl} \text { in leech's blood, } \\
\text { mgrms } \%\end{array}$} & \multirow{2}{*}{$\begin{array}{c}\text { Difference } \\
(\%)\end{array}$} \\
\hline & & Nos. of leeches & Nos. of & mples & \\
\hline \multirow{4}{*}{$\begin{array}{c}\text { No. } 4 \\
(1.950)\end{array}$} & \multirow{4}{*}{473} & No. 7 & $\begin{array}{l}\text { No. } 1 \\
\text { No. } 2 \\
\text { No. } 3\end{array}$ & $\begin{array}{l}486 \\
482 \\
446\end{array}$ & $\begin{array}{r}3.1 \\
-\quad 1.7 \\
-\quad 5.7\end{array}$ \\
\hline & & No. 8 & $\begin{array}{l}\text { No. } 1 \\
\text { No. } 2 \\
\text { No. } 3\end{array}$ & $\begin{array}{l}441 \\
450 \\
446\end{array}$ & $\begin{array}{r}-6.8 \\
-\quad 4.9 \\
-\quad 5.7\end{array}$ \\
\hline & & No. 9 & $\begin{array}{l}\text { No. } 1 \\
\text { No. } 2\end{array}$ & $\begin{array}{l}423 \\
428\end{array}$ & $\begin{array}{r}-10.1 \\
-9.5\end{array}$ \\
\hline & & No. 10 & $\begin{array}{l}\text { No. } 1 \\
\text { No. } 2 \\
\text { No. } 3\end{array}$ & $\begin{array}{l}410 \\
428 \\
432\end{array}$ & $\begin{array}{r}-13.6 \\
-\quad 9.5 \\
-\quad 8.7\end{array}$ \\
\hline & & & & $\operatorname{age}$ & $\pm 4.6 \%$ \\
\hline
\end{tabular}

Tables I and II give the comparison of the blood chlorine content of rabbits' blood between the direct and the indirect (leech) method.

A glance at these two tables will show that the error was almost exclusively in the negative sense (Cf. Table II* and III). Table III shows the same fact.

\section{TABLE III.}

Application of the leech method to clinical cases.

( 0.13 c.c. blood used for each sample for the determination of $\mathrm{NaCl}$ )

\begin{tabular}{|c|c|c|c|c|c|}
\hline \multirow{3}{*}{ Cases } & \multirow{3}{*}{$\begin{array}{l}\text { Diagnosis } \\
\quad \text { (Age) }\end{array}$} & \multicolumn{3}{|c|}{ Blood $\mathrm{NaCl}$ content in mgrus $\%$} & \multirow{3}{*}{$\begin{array}{c}\text { Difference } \\
(\%)\end{array}$} \\
\hline & & \multirow{2}{*}{$\begin{array}{c}\text { Direct } \\
\text { method } \\
\text { (auricular) }\end{array}$} & \multicolumn{2}{|c|}{ Indirect method } & \\
\hline & & & $\begin{array}{l}\text { Nos. of } \\
\text { leeches }\end{array}$ & $\begin{array}{l}\text { Nos. of } \\
\text { samples }\end{array}$ & \\
\hline \multirow{2}{*}{ No. 1} & \multirow{2}{*}{$\begin{array}{l}\text { Hydrocephalus internus } \\
\text { (13 years) }\end{array}$} & \multirow{2}{*}{442} & No. 1 & $\begin{array}{ll}\text { No. } 1 & 446 \\
\text { No. } 2 & \text { lost }\end{array}$ & $+0.4 *$ \\
\hline & & & No. 2 & $\begin{array}{ll}\text { No. } 1 & 437 \\
\text { No. } 2 & 428\end{array}$ & $\begin{array}{l}-1.1 \\
-3.2\end{array}$ \\
\hline No. 2 & $\begin{array}{l}\text { Jaundice (hepato-lienal } \\
\text { disease ( } 12 \text { years) }\end{array}$ & 458 & No. 3 & $\begin{array}{ll}\text { No. } 1 & 423 \\
\text { No. } 2 & 437 \\
\text { No. } 3 & 455 \\
\text { No. } 4 & 446 \\
\text { No. } 5 & 423\end{array}$ & $\begin{array}{l}-7.6 \\
-4.6 \\
-0.3 \\
-2.6 \\
-7.6\end{array}$ \\
\hline No. 3 & Pericarditis (15 jear's) & 456 & No. 4 & $\begin{array}{ll}\text { No. } 1 & 441 \\
\text { No. } 2 & 432 \\
\text { No. } 3 & 414 \\
\text { No. } 4 & 428 \\
\text { No. } 5 & 432\end{array}$ & $\begin{array}{l}-3.3 \\
-5.3 \\
-9.2 \\
-6.1 \\
-5.3\end{array}$ \\
\hline
\end{tabular}




\begin{tabular}{|c|c|c|c|c|c|c|}
\hline \multirow{3}{*}{ Cases } & \multirow{3}{*}{$\begin{array}{l}\text { Diagnosis } \\
\text { (Age) }\end{array}$} & \multicolumn{4}{|c|}{ Blood $\mathrm{NaCl}$ content in mgrms $\%$} & \multirow{3}{*}{$\begin{array}{c}\text { Difference } \\
(\%)\end{array}$} \\
\hline & & \multirow{2}{*}{$\begin{array}{c}\text { Direct } \\
\text { method } \\
\text { (auricular) }\end{array}$} & \multicolumn{3}{|c|}{ Indirect method } & \\
\hline & & & $\begin{array}{l}\text { Nos. of } \\
\text { leeches }\end{array}$ & $\begin{array}{l}\text { Nos. o } \\
\text { sample }\end{array}$ & & \\
\hline \multirow{4}{*}{ No. 4} & \multirow{4}{*}{ Typhoid fever (10 years) } & \multirow{4}{*}{453} & No. 5 & $\begin{array}{ll}\text { No. } 1 & 4 \\
\text { No. } 2 & 4 \\
\text { No. } 3 & 4\end{array}$ & $\begin{array}{l}468 \\
410 \\
437\end{array}$ & $\begin{array}{l}+1.1^{*} \\
-9.5 \\
-3.5\end{array}$ \\
\hline & & & No. 6 & $\begin{array}{ll}\text { No. } 1 & 4 \\
\text { No. } 2 & 4 \\
\text { No. } 3 & 4\end{array}$ & $\begin{array}{l}419 \\
410 \\
437\end{array}$ & $\begin{array}{l}-7.5 \\
-9.5 \\
-3.5\end{array}$ \\
\hline & & & No. 7 & $\begin{array}{ll}\text { No. } 1 & 4 \\
\text { No. } 2 & 4 \\
\text { No. } 3 & 4\end{array}$ & $\begin{array}{l}419 \\
432 \\
419\end{array}$ & $\begin{array}{l}-7.5 \\
-4.6 \\
-7.5\end{array}$ \\
\hline & & & No. 8 & $\begin{array}{ll}\text { No. } 1 & 4 \\
\text { No. } 2 & 4 \\
\text { No. } 3 & 4\end{array}$ & $\begin{array}{l}428 \\
437 \\
432\end{array}$ & $\begin{array}{l}-5.5 \\
-3.5 \\
-6.6\end{array}$ \\
\hline
\end{tabular}

Conclusion.

The leech method for the purpose of micro-analysis can be applied to blood chloride also. Here the error is mostly in the negative sense. 